tion pour la Recherche Médicale, the European Union, Institut National du Cancer, and Agence Nationale pour la Recherche.

Address correspondence to: Guido Kroemer, INSERM, U848, Institut Gustave Roussy, Pavillon de Recherche 1, 39 rue Camille-Desmoulins, F-94805 Villejuif, France. Phone: 33-1-42-11-60-46; Fax: 331-42-11-60-47; E-mail: kroemer@igr.fr.

1. Bowie, A.G., and Unterholzner, L. 2008. Viral evasion and subversion of pattern-recognition receptor signalling. Nat. Rev. Immunol. 8:911-922.

2. Luo, J., Solimini, N.L., and Elledge, S.J. 2009. Principles of cancer therapy: oncogene and non-oncogene addiction. Cell. 136:823-837.

3. Alexandroff, A.B., Jackson, A.M., O’Donnell, M.A., and James, K. 1999. BCG immunotherapy of bladder cancer: 20 years on. Lancet. 353:1689-1694.

4. Papadavid, E., Stratigos, A.J., and Falagas, M.E. 2007. Imiquimod: an immune response modifier in the treatment of precancerous skin lesions and skin cancer. Expert Opin. Pharmacother. 8:1743-1755.

5. Takeuchi, O., and Akira, S. 2008. MDA5/RIG-I and virus recognition. Curr. Opin. Immunol. 20:17-22.
6. Besch, R., et al. 2009. Proapoptotic signaling induced by RIG-I and MDA- 5 results in type I interferon-independent apoptosis in human melanoma cells. J. Clin. Invest. 119:2399-2411.

7. Hirabayashi, K., et al. 1999. Inhibition of cancer cell growth by polyinosinic-polycytidylic acid/cationic liposome complex: a new biological activity. Cancer Res. 59:4325-4333

8. Salaun, B., Coste, I., Rissoan, M.C., Lebecque, S.J., and Renno, T. 2006. TLR3 can directly trigger apoptosis in human cancer cells. J. Immunol. 176:4894-4901.

9. Salaun, B., Lebecque, S.J., Matikainen, S., Rimoldi, D., and Romero, P. 2007. Toll-like receptor 3 expressed by melanoma cells as a target for therapy? Clin. Cancer Res. 13:4565-4574.

10. McAllister, C.S., and Samuel, C.E. 2009. The RNAactivated protein kinase enhances the induction of interferon-beta and apoptosis mediated by cytoplasmic RNA sensors. J. Biol. Chem. 284:1644-1651.

11. Szczepanski, M.J., et al. 2009. Triggering of Tolllike receptor 4 expressed on human head and neck squamous cell carcinoma promotes tumor development and protects the tumor from immune attack. Cancer Res. 69:3105-3113.

12. Green, D.R., Ferguson, T., Zitvogel, L., and Kroemer, G. 2009. Immunogenic and tolerogenic cell death. Nat. Rev. Immunol. 9:353-363.

13. Pan, M., et al. 2009. Apoptosis induced by synthetic retinoic acid CD437 on human melanoma A375 cells involves RIG-I pathway. Arch. Dermatol. Res. 301:15-20.

14. Poeck, H., et al. 2008. 5'-Triphosphate-siRNA: turning gene silencing and Rig-I activation against melanoma. Nat. Med. 14:1256-1263.

15. Zitvogel, L., et al. 2008. The anticancer immune response: indispensable for therapeutic success? J. Clin. Invest. 118:1991-2001.

16. Andre, F., et al. 2006. Toll like receptor 3 expression and efficacy of adjuvant treatment with polyadenylic-polyuridylic acid in patients with axillary node positive breast cancer: results from two randomized trials. Presented at the 2006 ASCO Annual Meeting. June 2-5. Atlanta, Georgia, USA.

17. Panaretakis, T., et al. 2009. Mechanisms of preapoptotic calreticulin exposure in immunogenic cell death. EMBO J. 28:578-590.

18. Apetoh, L., Tesniere, A., Ghiringhelli, F., Kroemer, G., and Zitvogel, L. 2008. Molecular interactions between dying tumor cells and the innate immune system determine the efficacy of conventional anticancer therapies. Cancer Res. 68:4026-4030.

19. Borg, C., et al. 2004. Novel mode of action of c-kit tyrosine kinase inhibitors leading to NK cell-dependent antitumor effects. J. Clin. Invest. 114:379-388.

20. Menard, C., et al. 2009. Natural killer cell IFNgamma levels predict long-term survival with imatinib mesylate therapy in gastrointestinal stromal tumor-bearing patients. Cancer Res. 69:3563-3569.

\title{
Reduced levels of neurotransmitter-degrading enzyme PRCP promote obesity
}

\author{
Richard D. Palmiter
}

Howard Hughes Medical Institute and Departments of Biochemistry and Genome Sciences, University of Washington, Seattle, Washington, USA.

\begin{abstract}
The level of neurotransmitters present in the synaptic cleft is a function of the delicate balance among neurotransmitter synthesis, recycling, and degradation. While much is known about the processes controlling neurotransmitter synthesis and release, the enzymes that degrade peptide neurotransmitters are poorly understood. A new study in this issue of the JCI reveals the important role of neuropeptide degradation in regulating obesity (see the related article beginning on page 2291). Wallingford et al. provide evidence that, in mice, the enzyme prolylcarboxypeptidase (PRCP) degrades $\alpha$-melanocyte-stimulating hormone ( $\alpha-\mathrm{MSH})$ to an inactive form that is unable to inhibit food intake. Their studies indicate that PRCP expression promotes obesity, while inhibitors of the enzyme counteract obesity.
\end{abstract}

Neurons in the arcuate region of the hypothalamus that make the polypeptide hormone precursor proopiomelanocortin (POMC) are very important for body weight regulation, as revealed by the dramatic development of obesity after selective

Conflict of interest: The author has declared that no conflict of interest exists.

Nonstandard abbreviations used: ACTH, adrenocorticotropic hormone; MC4R, melanocortin 4 receptor; $\alpha$-MSH, $\alpha$-melanocyte-stimulating hormone; POMC, proopiomelanocortin; PRCP, prolylcarboxypeptidase.

Citation for this article: J. Clin. Invest. 119:2130-2133 (2009). doi:10.1172/JCI40001. inactivation of the neuronal Pomc gene in mice (1) and the presence of POMC mutations in humans with severe early-onset obesity (2). POMC is proteolytically processed into a number of physiologically important peptides, including endorphins, adrenocorticotropic hormone (ACTH), and $\alpha$-melanocyte-stimulating hormone $(\alpha-\mathrm{MSH})$. The loss of $\alpha-\mathrm{MSH}$ production by POMC-deficient neurons is primarily responsible for the resultant obesity (3). The 13 -amino-acid peptide $\alpha$-MSH (referred to as $\left.\alpha-\mathrm{MSH}_{1-13}\right)$ acts on the postsynaptic melanocortin 4 receptor (MC4R) in many brain regions to suppress appetite and stimulate metabolism (Figure 1). Failure of $\alpha-\mathrm{MSH}_{1-13}$ signaling due to mutations in the MC4R gene represents the most common form of genetically inherited human obesity (4).

The activity of POMC neurons and the synthesis of POMC are stimulated by hormones such as leptin and insulin and inhibited by fasting (5-7). Signaling by POMC neurons is kept in check by neighboring neurons that make neuropeptide $\mathrm{Y}$ (NPY), agouti-related protein (AgRP), and GABA (Figure 1). The hormones and neurotransmitters that regulate these two populations of hypothalamic neurons have been studied extensively during the last 15 years $(5-7)$. Whereas the events that lead up to activation of MC4R by $\alpha-\mathrm{MSH}_{1-13}$ are well known, virtually nothing is known about how $\alpha-\mathrm{MSH}_{1-13}$ activity is terminated. The extent of MC4R activation, and hence the extent of appetite loss, will be determined by the rate of $\alpha-\mathrm{MSH}_{1-13}$ release from POMC neurons and the rate at which it is degraded or otherwise cleared from the synaptic space. A seminal paper 
A

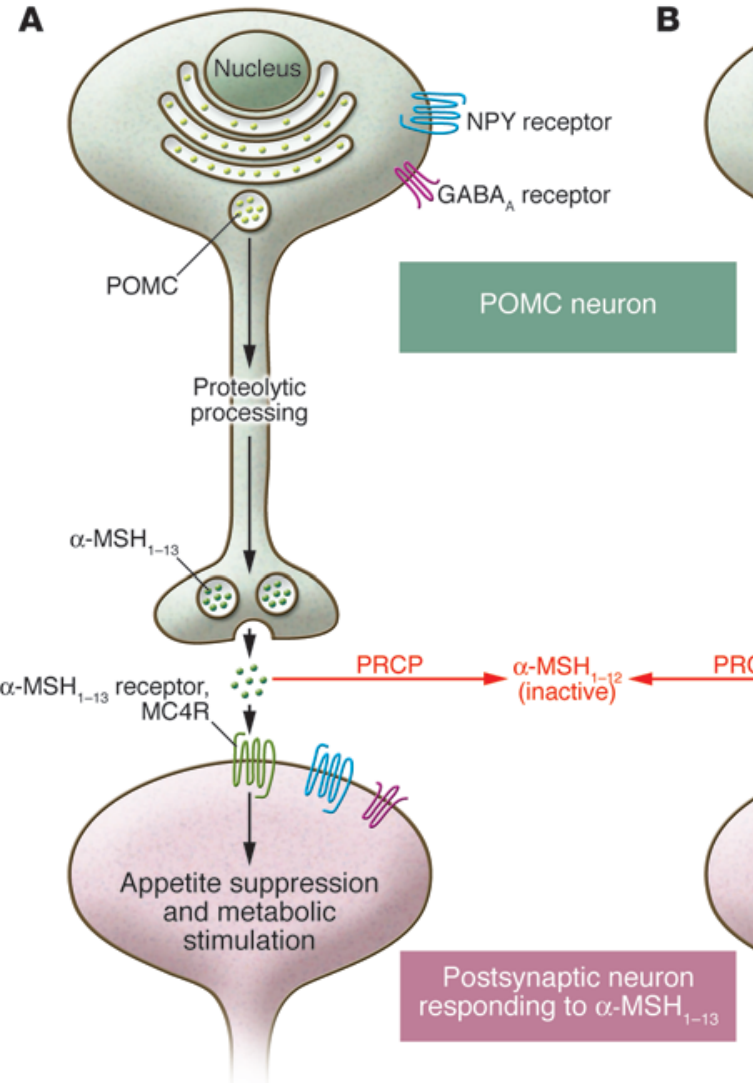

B

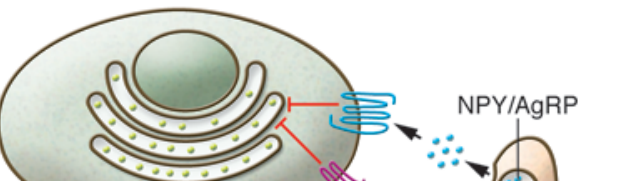




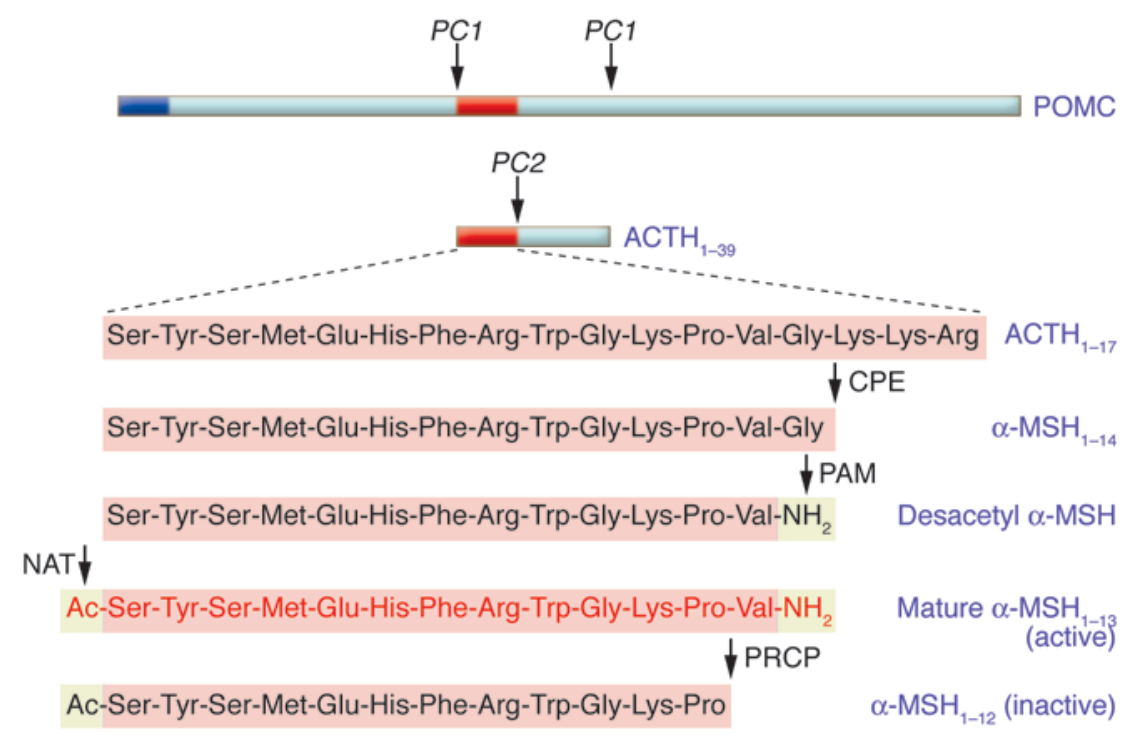

Figure 2

Processing of POMC to $\alpha-M S H$. POMC is synthesized in brain, pituitary gland, and skin cells. It is transported into the secretory pathway and processed by several enzymes into a variety of active peptides, depending on the cells in which it is synthesized. In hypothalamic neurons, POMC processing to produce $\alpha-\mathrm{MSH}$ is particularly important. The POMC precursor is first cut by proconvertase $1(\mathrm{PC} 1)$ to release $\mathrm{ACTH}_{1-39}$, which is then cut by proconvertase $2(\mathrm{PC} 2)$ to produce $\alpha-\mathrm{MSH}_{1-17}$. Carboxypeptidase $\mathrm{E}$ (CPE) removes the basic amino acids (Lys-Lys-Arg) from the $\mathrm{C}$ terminus, and peptidyl $\alpha$-amidating monooxygenase (PAM) converts the $\mathrm{C}$-terminal glycine to an amide $\left(\mathrm{NH}_{2}\right)$. The $\mathrm{N}$ terminus of $\alpha-\mathrm{MSH}_{1-13}$ is acetylated by $\mathrm{N}$-acetyltransferase (NAT) to produce mature, functional $\alpha-\mathrm{MSH}_{1-13}$. After release from synaptic vesicles, $\alpha-\mathrm{MSH}_{1-13}$ is inactivated (to $\alpha-\mathrm{MSH}_{1-12}$ ) by the action of PRCP, the subject of the Wallingford et al. (8) study in this issue of the $\mathrm{JCl}$.

to activate the coagulation-related protein prekallikrein - an early step in the production of the vasodilator bradykinin (14). The current study by Wallingford et al. (8) shows that this enzyme is also expressed in the brain, where it plays an important role in determining neuropeptide levels. Their studies began with a C57BL/6 mouse strain that carries a small region of chromosome 7 from a BALB/c strain. Those mice were significantly leaner compared with $\mathrm{C} 57 \mathrm{BL} / 6$ mice without the BALB/c chromosomal segment (15), prompting further investigation as to the gene(s) in that chromosomal interval that contribute to the lean phenotype. Further studies determined that the relevant BALB/c region on chromosome 7 includes only 4 protein-encoding genes; of those genes, Prcp was the likely candidate, given previous reports of a link between PRCP and metabolic syndrome (16). Sequencing revealed no polymorphisms within any of the coding regions of the 4 genes, but there was a single-base polymorphism at -718 in the presumptive promoter/enhancer region of the Prcp gene that was associated with a significant reduction in the level of Prcp mRNA in the brain of the mice carrying the $\mathrm{BALB} / \mathrm{c}$ allele. The authors also generated a complete knockout of the Prcp gene from mouse embryonic stem cells in which a lac $Z$ gene had randomly inserted into the fourth intron of the Prcp gene in a gene-trapping experiment. The Prcp-null $\left(\operatorname{PrcP}^{-/-}\right)$mice ate less and had even less fat than the mice with partial loss of the enzyme; furthermore, these animals were resistant to dietinduced obesity. The authors convincingly demonstrate that $\alpha-\mathrm{MSH}_{1-13}$ is a substrate for PRCP, which is known to remove the carboxyterminal residue from proteins with a penultimate proline residue. As predicted, the $\mathrm{Prcp}^{-/-}$mice had elevated levels of $\alpha-\mathrm{MSH}_{1-13}$ in the brain. Injection of $\alpha-\mathrm{MSH}_{1-13}$ into the brain of wild-type mice inhibited feeding, whereas injection of the same amount of the truncated, inactive $\alpha-\mathrm{MSH}_{1-12}$ was ineffective at reducing food consumption. As a final demonstration of the importance of PRCP, the authors showed that delivery of 2 different specific inhibitors of PRCP to the brain could suppress food intake by hungry mice in an
MC4R-dependent manner. Overall, this is a very satisfying set of results that logically leads from the identification of a candidate disease gene to determination of its mechanism of action.

\section{PRCP is expressed in the hypothalamus}

Wallingford et al. (8) show that many cells in the brain make PRCP, including cells in the hypothalamus, where $\alpha-\mathrm{MSH}_{1-13}$ is released onto postsynaptic neurons bearing MC4 receptors. PRCP has a signal peptide to direct it into the secretory pathway. Although PRCP was originally described as a lysosomal enzyme (17), it seems likely that its action on its known substrates, including $\alpha-\mathrm{MSH}_{1-13}$, occurs extracellularly. So, PRCP may be poised in the synaptic space to degrade $\alpha-\mathrm{MSH}_{1-13}$ and thereby reduce $\alpha-\mathrm{MSH}_{1-13}$ action, which would promote anorexia. It is also possible that the amount of PRCP in the extracellular space can be regulated; e.g., under starvation conditions, the activity of PRCP may be elevated in order to limit $\alpha-\mathrm{MSH}_{1-13}$ signaling and thereby help promote feeding and reduce metabolism. The converse situation has been demonstrated by studies showing that acetylation of desacetyl $\alpha-\mathrm{MSH}_{1-13}$ increases in response to the adiposity hormone leptin $(17,18)$, which stabilizes $\alpha-\mathrm{MSH}_{1-13}$ and thereby promotes weight loss.

\section{Future directions}

The present study adds an important new dimension to our understanding of how neuropeptide signaling is controlled in the brain. Moreover, it raises many intriguing questions and possibilities. Considering that $\alpha-\mathrm{MSH}_{1-13}$ also functions in the skin to control pigmentation via activation of melanocortin-1 receptors, changes in PRCP levels may also affect skin and hair color. Because PRCP is widely expressed at low levels in the brain and in the periphery, it is likely that there are additional substrates with penultimate proline residues in their sequence whose levels are controlled by PRCP. Consequently, the phenotype of the $\mathrm{Prcp}^{-/-}$mice may be more complex, and more interesting, than currently envisioned. For example, we can already anticipate that the levels of angiotensin II and bradykinin will be affected. Thus, there is much more to learn from the $\mathrm{PrCp}^{-/-}$mice generated by Wallingford and colleagues (8). It will also be interesting to determine whether mutations in the human PRCP 
gene are correlated with body weight. If it turns out that $\alpha-\mathrm{MSH}_{1-13}$ is the principal substrate of PRCP, then pharmacological inhibition of its action in the brain could help reduce appetite and weight gain; Wallingford et al. (8) demonstrated the efficacy of this strategy by injecting inhibitors directly into the brain, but brain-penetrating drugs would need to be developed to be clinically useful.

Address correspondence to: Richard D. Palmiter, Department of Biochemistry and Genome Sciences, Health Sciences Building, Room J-661F, Box 357370, University of Washington, 1959 Pacific Avenue, Seattle, Washington 98195, USA. Phone: (206) 543-6064; Fax: (206) 543-0858; E-mail: palmiter@u.washington.edu.

1. Smart, J.L., Tolle, V., and Low, M.J. 2006. Glucocorticoids exacerbate obesity and insulin resistance in neuron-specific proopiomelanocortin-deficient mice. J. Clin. Invest. 116:495-505.
2. Krude, H., et al. 1998. Severe early-onset obesity, adrenal insufficiency and red hair pigmentation caused by POMC mutations in humans. Nat. Genet. 19:155-157.

3. Coll, A.P., et al. 2004. Proopiomelanocortin and energy balance: insights from human and murine genetics. J. Clin. Endocrinol. Metab. 89:2557-2562.

4. Faroogi, I.S. 2008. Monogenetic human obesity. Front. Horm. Res. 36:1-11.

5. Saper, C.B., Chou, T.C., and Elmquist, J.K. 2002. The need to feed: homeostatic and hedonic control of eating. Neuron. 36:199-211.

6. Cone, R.D. 2005. Anatomy and regulation of the central melanocortin system. Nat. Neurosci. 8:571-578.

7. Morton, G.J., Cummings, D.E., Baskin, D.G., Barsh, G.S., and Schwartz, M.W. 2006. Central nervous system control of food intake and body weight. Nature. 443:289-295.

8. Wallingford, N., et al. 2009. Prolylcarboxypeptidase regulates food intake by inactivating $\alpha-\mathrm{MSH}$ in rodents. J. Clin. Invest. 119:2291-2303.

9. Prichard, L.E., Turnbull, A.V., and White, A. 2002. Pro-opiomelanocortin processing in the hypothalamus: impact on melanocortin signaling and obesity. J. Endocrinol. 172:411-421.

10. Jackson, R.S., et al. 1997. Obesity and impaired prohormone processing associated with mutations in the human prohormone convertase 1 gene. Nat. Genet. 16:303-306.

11. Rouillé, Y., et al. 1995. Proteolytic processing mechanisms in the biosynthesis of neuroendocrine peptides: the subtilisin-like proprotein convertases. Front. Neuroendocrinol. 16:322-361.

12. Naggert, J.K., et al. 1995. Hyperproinsulinaemia in obese fat/fat mice associated with a carboxypeptidase E mutation which reduces enzyme activity. Nat. Genet. 10:135-142.

13. Skidgel, R.A., and Erdos, E.G. 1998. Cellular carboxypeptidases. Immunol. Rev. 161:129-141.

14. Schmaier, A.H. 2003. The kallikrein-kinin and the renin-angiotensin systems have a multilayered interaction. Am. J. Physiol. Regul. Integr. Comp. Physiol. 285:R1-R13.

15. Diament, A.L., and Warden, C.H. 2004. Multiple linked mouse chromosome 7 loci influence body fat mass. Int. J. Obes. Relat. Metab. Disord. 28:199-210.

16. McCarthy, J.J., et al. 2003. Evidence for substantial effect modification by gender in a large-scale genetic association study of the metabolic syndrome among coronary heart disease patients. Hum. Genet. 114:87-98.

17. Wilkinson, C.W. 2006. Roles of acetylation and other post-translational modifications in melanocortin function and interactions with endorphins. Peptides. 27:453-471.

18. Guo, L., Munzberg, H., Stuart, R.C., Nillni, E.A., and Bjorbaek, C. 2004. N-acetylation of hypothalamic alpha-melanocyte-stimulating hormone and regulation by leptin. Proc. Natl. Acad. Sci. U. S. A. 101:11797-11802.

\title{
Engaging the lysosomal compartment to combat B cell malignancies
}

\author{
Kirsten Grønbæk ${ }^{1}$ and Marja Jäättelä ${ }^{2}$ \\ ${ }^{1}$ Department of Hematology, Rigshospitalet, Copenhagen, Denmark. ${ }^{2}$ Apoptosis Department and Centre for Genotoxic Stress Response, \\ Institute for Cancer Biology, Danish Cancer Society, Copenhagen, Denmark.
}

The combination of rituximab, a type I anti-CD20 $\mathrm{mAb}$, with conventional chemotherapy has significantly improved the outcome of patients with B cell malignancies. Regardless of this success, many patients still relapse with therapy-resistant disease, highlighting the need for the development of mAbs with higher capacity to induce programmed cell death. The so-called type II anti-CD20 mAbs (e.g., tositumomab) that trigger caspase-independent $B$ cell lymphoma cell death in vitro and show superior efficacy as compared with rituximab in eradicating target cells in mouse models are emerging as the next generation of therapeutic anti-CD20 mAbs. In this issue of the JCI, Ivanov and colleagues identify the lysosomal compartment as a target for type II mAbs (see the related article beginning on page 2143). These data encourage the further clinical development of type II mAbs as well as other lysosome-targeting drugs in the treatment of $B$ cell malignancies.

Caspase-mediated apoptosis is the main mechanism of action of most current anticancer treatments. Defects in apoptosis signaling pathways are, however, among the

Conflict of interest: The authors have declared that no conflict of interest exists.

Nonstandard abbreviations used: Ag, antigen.

Citation for this article: J. Clin. Invest. 119:2133-2136 (2009). doi:10.1172/JCI40259. major hallmarks of cancer, and apoptosisinducing therapies further select for highly apoptosis-refractory tumor cell clones (1). Accordingly, new strategies to kill cancer cells by nonapoptotic mechanisms have flourished during the past decade, and many mediators of alternate cell death pathways have been identified. Among them are the lysosomes with their large arsenal of proteolytic and lipolytic hydrolases $(2,3)$.
The lysosome: an emerging target for cancer therapy

Lysosomes function as cellular recycling and waste disposal units by degrading organelles and macromolecules delivered to the lysosomal compartment by autophagy, endocytosis, and phagocytosis (4). The degradation is performed by over 50 lysosomal hydrolases that can process all the major macromolecules of the cell to break down products available for metabolic reutilization. The lysosomal cell death pathways are characterized by partial lysosomal membrane permeabilization and the subsequent translocation of lysosomal hydrolases into the cytosol (2, 3). Once in the cytosol, lysosomal hydrolases, particularly cathepsin proteases, can trigger caspase-independent and BCL-2-insensitive cell death pathways even in highly apoptosisresistant cancer cells.

The potential of lysosomes as powerful "cell suicide bags" was recognized already in the 1950s by Christian de Duve, who received the Nobel prize for his discovery of 